\title{
Konservatorium der Gefühle
}

\section{Zur Musikauffassung in W.H. Wackenroders >Das merkwürdige musikalische Leben des Tonkünstlers Joseph Berglingerı}

Franziska Frühauf

Musik ist Gefühl und Gefühl ist Musik. Eine Platitüde, könnte man geneigt sein zu sagen. Dem Tonkünstler Joseph Berglinger allerdings wird diese seine Auffassung von Beruf und Berufung zum Verhängnis. Er ist ein Vorkämpfer der vielen romantischen Künstlergestalten, die sich - selber Produkte der dichterischen Phantasie - mit ihrem Verständnis von Kunst und Künstlertum von einer steril gewordenen, entzauberten Welt absetzen.

Berglingers Name sucht man vergeblich im Musiklexikon; seine Vita findet sich als letzter Eintrag in einem Büchlein, das 1796 unter dem Titel >Herzensergießungen eines kunstliebenden Klosterbruders anonym erschienen ist. ${ }^{1}$ In dieser literarischen Figur aber gipfelt eine Musikauffassung, welche im ganzen 18. Jahrhundert hoch wirksam war: die Vorstellung einer unmittelbaren Wechselbeziehung zwischen menschlichen Affekten und Musik.

\section{Von der Affektenlehre zur Gefühlsästhetik}

Die musikalische Affektenlehre, die tragendes Konzept barocker Kompositionskunst war, geht den Entsprechungen zwischen seelischen Leidenschaften und der Musik nach und nimmt damit eine bereits in der Antike verbreitete Bewertung der Musik auf. Sie ist einer Nachahmungsästhetik verpflichtet, die glaubt, durch die Imitation innerer Seelenzustände diese auch im Hörer lebendig werden zu lassen. Die Musik vermag die Bewegungen der Seele nämlich nicht nur mimetisch nachzubilden, sondern auch zu erzeugen: Dank der vielfältigen Systematik der sogenannten Musica Poetica kann der Affektgehalt von Texten musikalisch umgesetzt und im Hörer vergegenwärtigt werden. Eine besondere Häufung von Dissonanzen und Chromatik beispielsweise stellt den Affekt des Schmerzes dar, untermalt Leidensrufe und lenkt den Hörvorgang so in die vom Klagegestus eines Textes vorgeschriebene Richtung (mesto). Für Freude stehen hingegen Dur, Konsonanz, hohe Tonlagen und ein schnelles Tempo (allegro). In detaillierten Tonartencharakteristiken werden den Tonarten ihre ganz spezifischen Affekteigenschaften zu-

\footnotetext{
${ }^{1}$ Heute ist man sich in der Forschung darin einig, dass sich hinter der Figur des Klosterbruders, dem fiktiven Verfasser der kunsttheoretischen Schriften, Wackenroder als Autor und Ludwig Tieck als dessen Herausgeber verbergen.
} 
gesprochen. Es geht um das bis heute umstrittene Problem, dass musikalische Werke gleicher Tonart in einem bestimmten Stil- und Ausdruckswert miteinander verwandt sind, etwa im Dämonischen und Gewaltigen des d-moll oder im frühlingshaft Strahlenden des EDur. Selbst die Musikinstrumente werden zu Affektdarstellungen systematisiert. Musik wirkt somit nicht etwa dadurch, dass sie den Hörer in irgendeine unbestimmte Stimmungslage versetzt; mittels entsprechend gewählter musikalischer Parameter ist dieser vielmehr ganz gezielt vom Affektgehalt der Musik (und des Textes) gelenkt. Die Affekte sind weitgehend intersubjektiv verstanden. Es geht um die Freude und den Schmerz.

Um die Mitte des 18. Jahrhunderts löst eine neue Ästhetik die starren kompositorischen Vorgaben der Affektenlehre ab und stellt statt der Allgemeinheit der Affekte die Individualität der Empfindungen in den Mittelpunkt. Nun sind nicht mehr typisierte emotionale Erregungszustände im Sinne von Affekten wichtig, sondern die Dynamik persönlich empfundener Gefühlsbewegungen, in facettenreichen Abstufungen von der heftigsten Erschütterung bis zur sanften Regung des Gemüts. Die Nachahmungsästhetik wandelt sich zur Ausdrucksästhetik der Empfindsamkeit und des Sturm und Drang. Die Mimesis gerät verschärft in eine Spannung mit dem schöpferischen Prinzip der Poiesis. Der geniale Künstler treibt die reiche Gefühlswelt seines Ich hin zum Ausdruck in seiner reinsten Form, zur Musik, und noch reiner: zur Instrumentalmusik.

Noch bevor der Blick überhaupt auf Joseph Berglinger gefallen ist: die Verbindung von Ohr und Herz ist spannungsreich und mitnichten trivial! Affekte können in doppelter Weise mit musikalischen Phänomenen korrespondieren, entweder zwischen Komponist und Komposition oder zwischen Musik und Musikhörer. Während der Akzent im Barock eher auf der rezeptiven Seite des Hörvorgangs liegt, bei dem sich aufgrund gezielter kompositorischer Kunstgriffe ganz bestimmte emotionale Wirkungen einstellen, verschiebt er sich später auf das Hervorbringen von Musik: Die im Komponisten waltenden unfassbaren und mächtigen Gefühle provozieren die Werke der Tonkunst. Wie geht ein Musiker, ein Tonkünstler, mit dieser Spannung um?

\section{Gestörte Sympathien}

Der Knabe Joseph wächst in einem eher kunstfeindlichen Umfeld auf, das sich ausschliesslich den Anforderungen des Alltags widmet. Sein Vater, "Doktor der Arzneigelehrsamkeit und in dürftigen Ver- 
mögensumständen« $(103)^{2}$, kann die Familie nach dem Tod der Mutter nur mit Mühe und Not ernähren. Auch Joseph ist für den Arztberuf bestimmt, den der dem Pietismus verpflichtete alte Berglinger für ausgesprochen nützlich hält. Der Knabe fühlt sich aber sehr viel mehr zur Musik, seiner »Hauptfreude« (105), hingezogen. Immer mehr zieht er sich in die Welt der Phantasie und der Töne zurück und flieht nach einem Zusammenstoss mit dem Vater in die nahe bischöfliche Residenz zu einem Verwandten, der ihm die Ausbildung zum Musiker ermöglicht.

Musik ist Gefühl und »die feinsten Falten und Biegungen der Töne drückten sich in seiner weichen Seele $a b$ « (107). Joseph ist fasziniert von ihrer emotionalen Macht, die das Herz hüpfen läßt und "seine Nerven mit leisen Schauern« (106) durchdringt. Das körperliche Erlebnis löst sich aber gleich wieder auf im affektgeladenen Verlauf der Töne, dem er sich hingibt, "als wäre sein Körper mit zur Seele geworden« (107). Manchmal tönt die Musik - ganz im Sinne der barocken Affektenlehre - so klar und intensiv, dass er sie "wie Worte« versteht. Dann aber entzieht sich ihre Wirkung wieder jeglicher Bestimmung und Typisierung als eine "wunderbare Mischung von Fröhlichkeit und Traurigkeit in seinem Herzen«. Es sind gerade diese gemischten Gefühle, »die keine Kunst geschickter ist auszudrücken als die Musik« (108). Der Komponist ist für den jungen Joseph ein Zauberer, der über das Reich der Gefühle gebietet und das Herz des Hörers zu formen vermag. Diese magische Gabe will er auch erlangen, um die Herzen zu berühren, ja sogar um über sie zu herrschen und sie buchstäblich nach seiner Pfeife tanzen zu lassen. Darum betet er zur heiligen Cäcilia:

»Möchtest du auf Harfensaiten

Meinen schwachen Finger leiten,

Daß Empfindung aus ihm quillt;

Daß mein Spiel in tausend Herzen

Laut Entzücken, süße Schmerzen,

beides hebt und wieder stillt. ...

Öffne mir der Menschen Geister,

Daß ich ihrer Seelen Meister

Durch die Kraft der Töne sei;

$\mathrm{Daß}$ mein Geist die Welt durchklinge,

Sympathetisch sie durchdringe,

Sie berausch in Phantasei!«(112).

Wenn Joseph im Zusammenhang mit dem musikalischen Effekt von Sympathie redet, so braucht er hier ein ästhetisches Modewort des ausgehenden 18. Jahrhunderts. Die Idee aus der Naturphilosophie,

\footnotetext{
${ }^{2}$ Die Seitenzahlen in Klammern beziehen sich auf die Ausgabe: W.H. Wackenroder/L. Tieck, Herzensergießungen eines kunstliebenden Klosterbruders, Nachwort von R. Benz, Stuttgart 1997.
} 
dass zwei Körper in einer verborgenen Übereinstimmung koinzidieren, ‘zusammen affiziert sein können, ermöglicht, von Autoren wie Schubart und Herder auf die Musikästhetik übertragen, emotionale Korrespondenzen und verführt Joseph dazu, Musik in absoluter Identität mit seinen Gefühlen zu verstehen. Er möchte Komponist werden, denn Komponieren ist für ihn die genaue Umkehr des Hörens. Rezeptionsästhetik geht in Produktionsästhetik über und die Musik selber wird zu einem emotional verbindlichen Medium.

Das >zweite Hauptstück ‘ der Lebensbeschreibung setzt einige Jahre später ein. Berglinger ist es inzwischen gelungen, in das glanzvolle musikalische Amt des Kapellmeisters aufzusteigen. Mit der Karriere sind all seine Wünsche in Erfüllung gegangen, er hat die »höchste Stufe des Glücks« (105) erlangt - und ist doch todunglücklich. Voller Qualen hat er sich erst mit der Technik und den Gesetzmässigkeiten der Tonkunst vertraut machen müssen, ehe er daran denken konnte, sein "Gefühl mit den Tönen zu handhaben" (116); er harrt aus, in der Hoffnung auf eine "herrliche Zukunft«. Vor allem aber leidet er darunter, dass sich das Publikum von seiner Musik und ihrem tiefen emotionalen Ausdruck durchaus nicht ansprechen lässt. Vielmehr sehen seine Hörer in ihr nur ein Mittel zur gesellschaftlichen Repräsentation und zur Unterhaltung. Kurz: »Die prächtige Zukunft ist eine jämmerliche Gegenwart geworden« (116). Obwohl Berglinger allmählich an sich selbst und seiner Berufung zu zweifeln beginnt, fasst er wieder Mut, als ihm schliesslich doch noch der Durchbruch gelingt und seine Musik auf die Herzen seiner Zuhörer $\mathrm{zu}$ wirken beginnt. Es ist in derselben Zeit, als sich der Zustand seines Vaters verschlechtert - und bald nach seinem erfolgreichen Auftritt wird er ans Sterbebett gerufen. Zwar kann er sich noch mit dem Vater versöhnen, aber der Schmerz über die eigene Nutzlosigkeit - die zugesandten Gelder wurden von einer Schwester heimlich unterschlagen - verzehrt ihn. Innerlich zerrissen, aber auf dem Höhepunkt seines Schaffens angelangt, komponiert er sein letztes Auftragswerk: eine Passionsmusik. Nach der gloriosen Aufführung befällt den völlig Ermatteten eine Nervenschwäche und er stirbt "nicht lange darauf in der Blüte seiner Jahre» (122).

Musik ist für Berglinger ambivalent, gespalten in die unterschiedlichen Erfahrungen als Hörer und als Komponist. Er träumt von einer Musik, die seine Emotionen adäquat umsetzt, sie aufbewahrt und vom Publikum entsprechend gehört und nachempfunden wird - und muss dabei immer wieder die Diskrepanz zwischen seinen Gefühlen und der musikalischen Wirkung erleben und erleiden. Die Musik scheint sich der Ineinssetzung mit dem Gefühl zu entziehen. Die musikalische Sympathie-Vorstellung gerät bei Berglinger in die Krise. 
Warum aber scheitert Berglinger als >Tonkünstler`? Immer wieder haben sich dies die Interpreten der Berglingerschen Vita gefragt. Und immer wieder wurde auf seine künstlerische Existenz der Verdacht des Dilettantismus erhoben. Wer Musik ausübend andauernd über Gefühle spricht, scheint in ästhetischer Hinsicht mehr als zweifelhaft. Diese Vermutung erhärtet sich spätestens dann, wenn man Hanslicks Formel Glauben schenkt, dass der Laie bei der Musik am meisten, der Künstler am wenigsten empfinde. - Solche Deutungsversuche befriedigen nicht, weichen Berglingers künstlerischer Problematik wahrscheinlich genauso aus wie eine Verteidigung oder Verherrlichung seiner Fähigkeiten.

Die treffendste Analyse bietet der Text selber. Zwischen Berglinger und uns Lesern steht nämlich als vermittelnde Instanz der >kunstliebende Klosterbruder`, der getreulich berichtende Gewährsmann und angebliche Verfasser der kleinen Schrift. Er steht Berglinger, seinem "innigsten Freund " (102), am nächsten und versucht als erster, das "merkwürdige musikalische Leben« $z u$ verstehen. In seiner Schlussbetrachtung bedauert er, "daß eben seine hohe Phantasie es sein mußte, die ihn aufrieb«. Er sieht die Diskrepanz von Kunstgenuss und Schaffenskraft sehr genau: »Soll ich sagen, daß er vielleicht mehr dazu geschaffen war, Kunst zu genießen als auszuüben?«. Und er fragt sich schliesslich, ob es nicht die grösste Herausforderung an einen Künstler sei, die "hohe Phantasie« nicht eigenmächtig zu verwalten, sondern »in dieses irdische Leben einweben ${ }^{3} \mathrm{zu}$ lassen, und ob es "etwas noch Wundervolleres, noch Göttlicheres als die Kraft der Phantasie« (123) gebe.

\section{Wandlung der Gefühle}

Wer ist dieser Klosterbruder? Er trägt kaum individuelle Züge, widmet sein Leben in der Grundhaltung frommer Kunstliebe ganz den "gebenedeiten Kunstheiligen" (5). Wackenroder hat sich mit dieser Figur wohl eine Maske aufgesetzt, die seinem eigenen KunstCredo verpflichtet ist.

Die in klösterlicher Einsamkeit geschriebenen Aufsätze sind unter dem Titel `Herzensergießungen`zusammengefasst. >Giesst ‘ auch der Klosterbruder seine Gefühle in Kunst >ein « wie sein Freund? (Vielleicht sind es gar Berglingers Gefühle, die in einer doppelten, sympathetisch motivierten Übermittlung an den Leser weitergegeben werden!) Oder heisst `Ergiessung‘, dass die Gefühle freigegeben werden und offen bleiben soll, was sie beim Leser bewirken? Ganz am Schluss bricht der Klosterbruder, von Erinnerungsschmerz über-

\footnotetext{
${ }^{3}$ Berglingers Biographie zeigt, wie prekär der Glaube an die künstlerische Autonomie ist, wie verhängnisvoll es ist, wenn Kunst und Leben in Spannung zueinander stehen - ein Motiv, das in der Literatur immer wieder begegnet.
} 
wältigt, ab: »Ich kann aber nach diesen Erinnerungen an meinen Joseph nichts mehr schreiben. - Ich beschließe mein Buch - und möchte nur, daß es einem oder dem anderen zur Erweckung guter Gedanken dienlich wäre. - «(123). Wollen die Gedankenstriche die "guten Gedanken« im Leser erzeugen? Rechnen sie mit Sympathie? Anders gefragt: Lassen sich Gefühle durch Phantasie aufbewahren, gewissermassen konservieren und im Herzen der Hörer (und Leser) reanimieren? Im von Wackenroder (oder Berglinger?) geschriebenen und von Tieck (oder dem Klosterbruder?) herausgegebenen musiktheoretischen Aufsatz über die >Wunder der Tonkunst< lautet eine zentrale Stelle:

"Es scheinen uns diese Gefühle, die in unserm Herzen aufsteigen, manchmal so herrlich und groß, daß wir sie wie Reliquien in kostbare Monstranzen einschließen, freudig davor niederknieen und im Taumel nicht wissen, ob wir unser eignes menschliches Herz oder ob wir den Schöpfer, von dem alles Große und Herrliche herabkommt, verehren.

$\mathrm{Zu}$ dieser Aufbewahrung der Gefühle sind nun verschiedene schöne Erfindungen gemacht worden, und so sind alle schönen Künste entstanden. Die Musik aber halte ich für die wunderbarste dieser Erfindungen, ... - weil sie eine Sprache redet, die wir im ordentlichen Leben nicht kennen, die wir gelernt haben, wir wissen nicht wo und wie, und die man allein für die Sprache der Engel halten möchte«"

Die Kunst, vornehmlich die Tonkunst, dient der Aufbewahrung der Gefuhle, behandelt diese sogar "wie Reliquien in kostbare[n] Monstranzen«. Dort aber sind sie eingeschlossen. Musik vermag sie nicht in ihrer subjektiven Prägung zu erhalten, sondern lagert sie aus in einen allgemeinen ästhetischen Raum, über den Berglinger auch als Komponist nicht mehr verfügt. Ihm wird schmerzlich bewusst, dass die absolute Identität von Musik und Gefühl, von der er als jugendlicher Musikfanatiker noch geträumt hat, nicht möglich ist: Einerseits verweigert sich die Musik mit ihren "zwingenden mathematischen Gesetze[n] « und ihrer »Kunstgrammatik» (116) immer wieder der völligen Emotionalisierung, andererseits ist ihm ein unmittelbarer Erfolg beim Publikum versagt. Er sieht sich beim Komponieren mit einer objektivierenden und mit einer verwandelnden Kraft konfrontiert, und beide Kräfte entreissen ihm die Macht über die eigenen Gefühle.

Gefühle lassen sich nicht aufbewahren, ohne dass sich zugleich die Möglichkeit ihrer Transformation eröffnet. So dient Musik zwar der Aufbewahrung, verwandelt aber zugleich die Gefühle - und entfremdet sie. Wackenroders frühromantische Musiktheorie unterbreitet eine Gefühlsästhetik, die sowohl über die Vorstellung der abbildenden Nachahmung als auch über das Ideal des reinen Ausdrucks

\footnotetext{
${ }^{4}$ W.H. Wackenroder, Die Wunder der Tonkunst, in: W.H. Wackenroder/L. Tieck, Phantasien über die Kunst, Stuttgart 1983, 67.
} 
hinausgeht. Musik vollzieht eine Metamorphose des Emotionalen, welche die Dimension bloss sympathetischer Erregung überschreitet. Darin ist sie »Sprache der Engel«.

\section{An die Musik ${ }^{5}$}

Musik: Atem der Statuen. Vielleicht:

Stille der Bilder. Du Sprache wo Sprachen

enden. Du Zeit, die senkrecht steht auf der Richtung vergehender Herzen.

Gefühle zu wem? O du der Gefühle

Wandlung in was? - : in hörbare Landschaft.

Du Fremde: Musik. Du uns entwachsener

Herzraum. Innigstes unser,

das, uns übersteigend, hinausdrängt, -

heiliger Abschied:

da uns das Innre umsteht

als geübteste Ferne, als andre

Seite der Luft:

rein,

riesig,

nicht mehr bewohnbar.

Franzisca Frühauf ist Assistentin am Lehrstuhl für Systematische Theologie bei Prof. Pierre Bühler.

${ }^{5}$ R.M. Rilke, Sämtliche Werke, hg. vom Rilke-Archiv, in Verb. mit R. Sieber-Rilke besorgt durch E. Zinn, Bd. 2, Wiesbaden 1955, 111. 